\title{
The Number of Fixed Points of AND-OR Networks with Chain Topology
}

\author{
Alan Veliz-Cuba \\ Lauren Geiser
}

University of Dayton, USA

received $2016-X X-X X$

AND-OR networks are Boolean networks where each coordinate function is either the AND or OR logical operator. We study the number of fixed points of these Boolean networks in the case that they have a wiring diagram with chain topology. We find closed formulas for subclasses of these networks and recursive formulas in the general case. Our results allow for an effective computation of the number of fixed points in the case that the topology of the Boolean network is an open chain (finite or infinite) or a closed chain.

Keywords: Boolean networks, fixed points, AND-OR networks, one-dimensional cellular automata

\section{Introduction}

Boolean networks, $f:\{0,1\}^{n} \rightarrow\{0,1\}^{n}$, have been used to study problems arising from areas such as mathematics, computer science, and biology (Akutsu et al., 1998, Albert and Othmer 2003; Mendoza and Xenarios, 2006, Jarrah et al. 2010, Veliz-Cuba and Stigler, 2011). A particular problem of interest is counting the number of fixed points ( $x$ such that $f(x)=x$ ). To simplify this problem one can restrict the class of Boolean functions or the topology of the network (Agur et al., 1988, Aracena et al. 2004, Jarrah et al. 2007, Aracena, 2008, Murrugarra and Laubenbacher, 2011; Veliz-Cuba and Laubenbacher, 2011; Jarrah et al. 2010; Veliz-Cuba et al. 2013, 2014, 2015, , which in some cases allows to find effective algorithms or formulas in closed form.

In this manuscript we focus on the number of fixed points of AND-OR networks (each Boolean function is either the AND or the OR operator) that have open or closed chain topology. We first consider the case of finite open chain topology and find a recursive formula (Theorem 2.6) and sharp lower and upper bounds. We then consider the case of infinite and closed chain topology, and show how they can be reduced to the case of finite open chain topology (Theorems 3.1 3.4.

\section{Open Chain}

Let $f=\left(f_{1}, \ldots, f_{n}\right):\{0,1\}^{n} \rightarrow\{0,1\}^{n}$ with $n \geq 2$ be an AND-OR network such that its wiring diagram is a chain, Fig 1 That is, we consider Boolean networks of the form:

$$
f_{1}=x_{2}, \quad f_{2}=x_{1} \diamond_{2} x_{3}, \quad f_{3}=x_{2} \diamond_{3} x_{4}, \quad \ldots \quad, \quad f_{n-1}=x_{n-2} \diamond_{n-1} x_{n}, \quad f_{n}=x_{n-1},
$$

ISSN subm. to DMTCS C 2016 by the author(s) Distributed under a Creative Commons Attribution 4.0 International License 


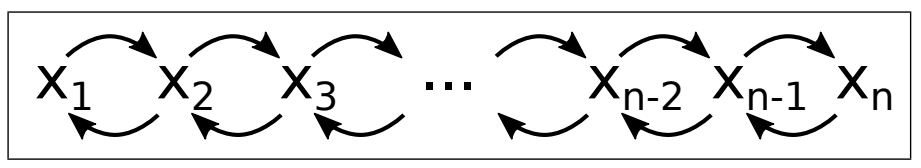

Fig. 1: Wiring diagram with open chain topology.

where $\diamond_{i}$ is the AND $(\wedge)$ or the OR $(\vee)$ operator.

Because this family of Boolean networks is completely determined by the sequence of logical operators $\diamond_{2}, \diamond_{3}, \ldots, \diamond_{n-1}$, we can use this sequence to represent the network. Furthermore, consecutive occurrences of the same logical operator can be denoted as $\wedge^{k}$ or $\vee^{k}$.

We are interested in the number of fixed points of such Boolean networks. For simplicity we denote the elements of $\{0,1\}^{n}$ as binary strings (omitting parentheses). Also, we will use the notation $\mathbf{0}=00 \cdots 0$ and $\mathbf{1}=11 \cdots 1$, where the length of the strings will be clear from the context. Note that $\mathbf{0}$ and $\mathbf{1}$ are fixed points of all AND-OR networks with chain topology.

Example 2.1. Our running example will be the AND-OR network

$$
\begin{aligned}
& f_{1}=x_{2}, \quad f_{2}=x_{1} \wedge x_{3}, \quad f_{3}=x_{2} \wedge x_{4}, \quad f_{4}=x_{3} \vee x_{5}, \quad f_{5}=x_{4} \wedge x_{6}, \quad f_{6}=x_{5} \vee x_{7}, \\
& f_{7}=x_{6} \vee x_{8}, \quad f_{8}=x_{7} \vee x_{9}, \quad f_{9}=x_{8} \wedge x_{10}, \quad f_{10}=x_{9} \wedge x_{11}, \quad f_{11}=x_{10} \vee x_{12}, \quad f_{12}=x_{11} .
\end{aligned}
$$

This network can be represented by the sequence of operators $\wedge \wedge \vee \wedge \vee \vee \vee \wedge \wedge \vee$. We can further simplify this representation to $\wedge^{2} \vee \wedge \vee^{3} \wedge^{2} \vee$. This AND-OR network has 13 fixed points listed in Table 1 (first column).

The next lemma states that the number of fixed points depends only on the powers of the operators. Since we do not know which operator is last $(\wedge$ or $\vee$ ), we will simply use ellipses without explicitly writing the last operator.

Lemma 2.2. The AND-OR networks $f=\wedge^{k_{1}} \vee^{k_{2}} \wedge^{k_{3}} \ldots$ and $g=\vee^{k_{1}} \wedge^{k_{2}} \bigvee^{k_{3}} \ldots$ have the same number of fixed points.

Proof: Consider $\phi:\{0,1\}^{n} \rightarrow\{0,1\}^{n}$ given by $\phi\left(x_{1}, \ldots, x_{n}\right)=\left(\neg x_{1}, \ldots, \neg x_{n}\right)$, where $\neg$ is the logical operator NOT. Using the fact that $\neg(p \wedge q)=\neg p \vee \neg q$ and $\neg(p \vee q)=\neg p \wedge \neg q$, it follows that $f(\phi(x))=\phi(g(x))$. Then, $x$ will be a fixed point of $g$ if and only if $\phi(x)$ is a fixed point of $f$. So, $\phi$ is a bijection between the fixed points of $g$ and $f$.

Because we are interested in the number of fixed points, we will simply use $\left(k_{1}, k_{2}, \ldots, k_{m}\right)$ to refer to a network. For instance, the AND-OR network seen in Example 2.1 can be represented simply by $(2,1,1,3,2,1)$. We denote the number of fixed points by $\mathcal{F}\left(k_{1}, k_{2}, \ldots, k_{m}\right)$.

The following lemma states that consecutive variables that have the same logical operator must be equal.

Lemma 2.3. Consider an AND-OR network $f$ represented by $\left(k_{1}, k_{2}, \ldots, k_{m}\right)$. Denote an element of the domain of $f$ by $\boldsymbol{x}=\left(\boldsymbol{x}^{1}, \boldsymbol{x}^{2}, \ldots, \boldsymbol{x}^{m}\right)$, where $\boldsymbol{x}^{1} \in\{0,1\}^{k_{1}+1}, \boldsymbol{x}^{m} \in\{0,1\}^{k_{m}+1}$, and $\boldsymbol{x}^{i} \in\{0,1\}^{k_{i}}$ for $i=2, \ldots, m-1$. If $\boldsymbol{x}$ is a fixed point of $f$, then $\boldsymbol{x}^{i}=\mathbf{0}$ or $\boldsymbol{x}^{i}=\boldsymbol{1}$ for $i=1, \ldots, m$.

Proof: Let $\mathbf{x}$ be a fixed point of $f$. We use $\left(\mathbf{x}^{i}\right)_{j}$ to denote the $j$-th coordinate of $\mathbf{x}^{i}$. Note that $\left(\mathbf{x}^{1}\right)_{1}=$ $\left(\mathbf{x}^{1}\right)_{2}$ and $\left(\mathbf{x}^{m}\right)_{k_{m}}=\left(\mathbf{x}^{m}\right)_{k_{m}+1}$ by definition of $f$ (the first and last coordinate functions of $f$ depend on single variables). 
Now, the rest of the proof follows from the fact that if $q=p \wedge r$ and $r=q \wedge s$ or if $q=p \vee r$ and $r=q \vee s$, then $q=r$. This implies that consecutive variables, $\left(\mathbf{x}^{i}\right)_{j}$ and $\left(\mathbf{x}^{i}\right)_{j+1}$, that have the same logical operators must be the same.

The next proposition states that the numbers $k_{i}$ in $\mathcal{F}\left(k_{1}, \ldots, k_{m}\right)$ can be assumed to be at most 2 for $2 \leq$ $i \leq m-1$, and 1 for $k_{1}$ and $k_{m}$. For example, this will imply that $\mathcal{F}(2,1,1,3,2,1)=\mathcal{F}(1,1,1,2,2,1)$ and $\mathcal{F}(2,5,3,1,4,3)=\mathcal{F}(1,2,2,1,2,1)$.

Example 2.1 (cont.) We highlight the structure of the fixed points of $\wedge^{2} \vee \wedge \vee^{3} \wedge^{2} \vee$ in Table 1 (second column).

Proposition 2.4. $\mathcal{F}\left(k_{1}, k_{2}, \ldots, k_{m-1}, k_{m}\right)=\mathcal{F}\left(1, \min \left\{k_{2}, 2\right\}, \ldots, \min \left\{k_{m-1}, 2\right\}, 1\right)$ for all positive integers $k_{i}$.

Proof: We will use the notation of Lemma 2.3.

We first show that $f=\wedge^{k_{1}} \vee^{k_{2}} \wedge^{k_{3}} \ldots$ and $g=\wedge \vee^{k_{2}} \wedge^{k_{3}} \ldots$ have the same number of fixed points. Let $\mathbf{x}=\left(\mathbf{x}^{1}, \ldots, \mathbf{x}^{m}\right)$ be a fixed point of $f$. Then, by Lemma 2.3 we have $\mathbf{x}^{1}=\mathbf{0}$ or $\mathbf{x}^{1}=\mathbf{1}$. Consider $\mathbf{y}=\left(\mathbf{z}, \mathbf{x}^{2}, \ldots, \mathbf{x}^{m}\right)$, where $\mathbf{z}=\left(\left(\mathbf{x}^{1}\right)_{1},\left(\mathbf{x}^{1}\right)_{2}\right)$. It can be checked that $\mathbf{y}$ is a fixed point of $g$. Now, if $\mathbf{y}=\left(\mathbf{z}, \mathbf{x}^{2}, \ldots, \mathbf{x}^{m}\right)$ is a fixed point of $g$, Lemma 2.3 implies that $\mathbf{z}=\mathbf{0}$ or $\mathbf{z}=\mathbf{1}$. We define $\mathbf{x}=\left(\mathbf{x}^{1}, \ldots, \mathbf{x}^{m}\right)$ in the domain of $f$, where $\mathbf{x}^{1}=\mathbf{0}$ if $\mathbf{z}=\mathbf{0}$ and $\mathbf{x}^{1}=\mathbf{1}$ if $\mathbf{z}=\mathbf{1}$. Then, it can be checked that $\mathbf{x}$ is a fixed point of $f$. This shows that $\mathcal{F}\left(k_{1}, k_{2}, \ldots, k_{m-1}, k_{m}\right)=\mathcal{F}\left(1, k_{2}, \ldots, k_{m-1}, k_{m}\right)$, and similarly it can be shown that $\mathcal{F}\left(1, k_{2}, \ldots, k_{m-1}, k_{m}\right)=\mathcal{F}\left(1, k_{2}, \ldots, k_{m-1}, 1\right)$.

We now show that for $k_{2} \geq 2, f=\wedge^{k_{1}} \vee^{k_{2}} \wedge^{k_{3}} \ldots$ and $g=\wedge^{k_{1}} \vee^{2} \wedge^{k_{3}} \ldots$ have the same number of fixed points. The general case is analogous. Let $\mathbf{x}=\left(\mathbf{x}^{1}, \mathbf{x}^{2}, \ldots, \mathbf{x}^{m}\right)$ be a fixed point of $f$. Then, by Lemma 2.3 we have $\mathbf{x}^{2}=\mathbf{0}$ or $\mathbf{x}^{2}=\mathbf{1}$. Consider $\mathbf{y}=\left(\mathbf{x}^{1}, \mathbf{z}, \mathbf{x}^{3}, \ldots, \mathbf{x}^{m}\right)$, where $\mathbf{z}=\left(\left(\mathbf{x}^{2}\right)_{1},\left(\mathbf{x}^{2}\right)_{2}\right)$. It can be checked that $\mathbf{y}$ is a fixed point of $g$. Now, if $\mathbf{y}=\left(\mathbf{x}^{1}, \mathbf{z}, \mathbf{x}^{3}, \ldots, \mathbf{x}^{m}\right)$ is a fixed point of $g$, Lemma 2.3 implies that $\mathbf{z}=\mathbf{0}$ or $\mathbf{z}=\mathbf{1}$. We define $\mathbf{x}=\left(\mathbf{x}^{1}, \mathbf{x}^{2}, \ldots, \mathbf{x}^{m}\right)$ in the domain of $f$, where $\mathbf{x}^{1}=\mathbf{0}$ if $\mathbf{z}=\mathbf{0}$ and $\mathbf{x}^{1}=\mathbf{1}$ if $\mathbf{z}=\mathbf{1}$. Then, it can be checked that $\mathbf{x}$ is a fixed point of $f$. This shows that $\mathcal{F}\left(k_{1}, k_{2}, \ldots, k_{m-1}, k_{m}\right)=\mathcal{F}\left(k_{1}, 2, k_{3}, \ldots, k_{m-1}, k_{m}\right)$ for $k_{2} \geq 2$.

Example 2.1 (cont.) Proposition 2.4 guarantees that $\wedge^{2} \vee \wedge \vee^{3} \wedge^{2} \vee$ and $\wedge \vee \wedge \vee^{2} \wedge^{2} \vee$ have the same number of fixed points. We can consider the second AND-OR network as a "reduced" version of the original AND-OR network. This is illustrated in Table 1 (third column).

Proposition 2.5. Let $r_{1}, \ldots, r_{m}$ in $\{1,2\}$, and $m \geq 2$. Then, we have the following

$$
\mathcal{F}\left(1, r_{1}, \ldots, r_{m}, 1\right)= \begin{cases}\mathcal{F}\left(1, r_{3}, \ldots, r_{m}, 1\right)+\mathcal{F}\left(r_{3}, \ldots, r_{m}, 1\right), & \text { for } r_{1}=1, r_{2}=1 \\ \mathcal{F}\left(2, r_{3}, \ldots, r_{m}, 1\right)+\mathcal{F}\left(1, r_{3}, \ldots, r_{m}, 1\right), & \text { for } r_{1}=1, r_{2}=2 \\ \mathcal{F}\left(1,1, r_{3}, \ldots, r_{m}, 1\right)+\mathcal{F}\left(r_{3}, \ldots, r_{m}, 1\right), & \text { for } r_{1}=2, r_{2}=1 \\ \mathcal{F}\left(1,2, r_{3}, \ldots, r_{m}, 1\right)+\mathcal{F}\left(1, r_{3}, \ldots, r_{m}, 1\right), & \text { for } r_{1}=2, r_{2}=2\end{cases}
$$

Proof: We will use the notation of Lemma 2.3 .

If $r_{1}=1, r_{2}=1$, then we claim that any fixed point of $f=\wedge \vee \wedge \vee^{r_{3}} \wedge^{r_{4}} \ldots$ is of the form $\mathbf{x}=\left(\mathbf{x}^{0}, \mathbf{x}^{1}, \mathbf{x}^{2}, \ldots, \mathbf{x}^{m}, \mathbf{x}^{m+1}\right)$ where either $\mathbf{x}^{0}=\mathbf{0}$ and $\mathbf{z}=\left(\mathbf{x}^{1}, \mathbf{x}^{2}, \ldots, \mathbf{x}^{m}, \mathbf{x}^{m+1}\right)$ is a fixed point of $g=\wedge \vee^{r_{3}} \wedge^{r_{4}} \ldots$ or $\mathbf{x}^{0}=\mathbf{x}^{1}=\mathbf{1}$ and $\mathbf{z}=\left(\mathbf{x}^{2}, \ldots, \mathbf{x}^{m}, \mathbf{x}^{m+1}\right)$ is a fixed point of $h=\vee^{r_{3}} \wedge^{r_{4}} \ldots$. 


\begin{tabular}{ccc}
\hline Fixed points & Structure from Lemma 2.3 & "Reduced" system (Proposition 2.4 ) \\
\hline 000000000000 & 000000000000 & 0000000000 \\
000000000011 & 000000000011 & 0000000011 \\
000001110000 & 000001110000 & 0000110000 \\
000001111111 & 000001111111 & 0000111111 \\
000001110011 & 000001110011 & 0000110011 \\
000111110000 & 000111110000 & 0011110000 \\
000111110011 & 000111110011 & 0011110011 \\
000111111111 & 000111111111 & 0011111111 \\
111100000000 & 111100000000 & 1110000000 \\
111100000011 & 111100000011 & 1110000011 \\
111111110000 & 111111110000 & 1111110000 \\
111111110011 & 111111110011 & 1111111111 \\
111111111111 & 111111111111 & \\
\hline
\end{tabular}

Tab. 1: Fixed points of the AND-OR network $\wedge^{2} \vee \wedge \vee^{3} \wedge^{2} \vee$. First column: fixed points. Second column: fixed points with the structure given by Lemma 2.3 highlighted. Third column: fixed points of reduced network, $\wedge^{2} \vee \wedge \vee^{2} \wedge^{2} \vee$, with the structure given by Lemma 2.3 highlighted.

Indeed, the system of Boolean equations for fixed points is

$$
\begin{aligned}
x_{1} & =x_{2} \\
x_{2} & =x_{1} \wedge x_{3} \\
x_{3} & =x_{2} \vee x_{4} \\
x_{4} & =x_{3} \wedge x_{5} \\
x_{5} & =x_{4} \vee x_{6} \\
& \vdots \\
x_{n} & =x_{n-1}
\end{aligned}
$$

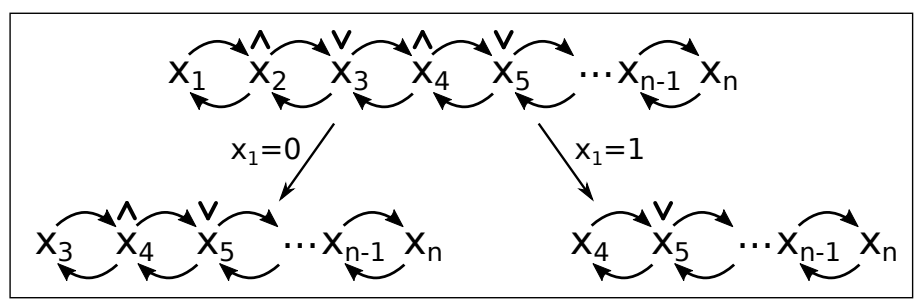

Fig. 2: Idea behind the proof of Proposition 2.5 (logical operators are included for clarity). Considering the cases $x_{1}=0$ and $x_{1}=1$ yields systems of equations that correspond to smaller AND-OR networks.

We divide this system of equations in the cases $x_{1}=0$ and $x_{1}=1$. Then, using the fact that $1=m \wedge n$ implies that $m=n=1$, that $0=m \vee n$ implies $m=n=0$, it follows that we obtain the two systems 


$$
\begin{aligned}
x_{3} & =x_{4} \\
x_{4} & =x_{3} \wedge x_{5} \\
x_{5} & =x_{4} \vee x_{6} \\
& \vdots \\
x_{n} & =x_{n-1}
\end{aligned}
$$

and

$$
\begin{aligned}
x_{4} & =x_{5} \\
x_{5} & =x_{4} \vee x_{6} \\
& \vdots \\
x_{n} & =x_{n-1},
\end{aligned}
$$

corresponding to the cases $x_{1}=0$ and $x_{1}=1$, respectively (see Fig. 2). This means that the number of fixed points of $f$ is equal to the number of solutions of these two systems. Since the solutions of the first system are the fixed points of $g=\wedge \vee^{r_{3}} \wedge^{r_{4}} \ldots$ and the solutions of the second system are the fixed points of $h=\vee^{r_{3}} \wedge^{r_{4}} \ldots$, we obtain $\mathcal{F}\left(1,1,1, r_{3} \ldots, r_{m}, 1\right)=\mathcal{F}\left(1, r_{3}, \ldots, r_{m}, 1\right)+\mathcal{F}\left(r_{3}, \ldots, r_{m}, 1\right)$.

The proof for the other three cases is similar.

By convention, we denote the AND-OR network $f\left(x_{1}, x_{2}\right)=\left(x_{2}, x_{1}\right)$ by an empty sequence, () . We also use the convention $\mathcal{F}\left(0, k_{1}, \ldots, k_{m}, 0\right)=\mathcal{F}\left(k_{1}, \ldots, k_{m}, 0\right)=\mathcal{F}\left(0, k_{1}, \ldots, k_{m}\right)=\mathcal{F}\left(k_{1}, \ldots, k_{m}\right)$ which will simplify the formulation of upcoming results.

Theorem 2.6. With the convention above, we have that for $m \geq 3$ and $k_{i} \geq 1$

$$
\mathcal{F}\left(k_{1}, \ldots, k_{m}\right)=\mathcal{F}\left(k_{2}-1, k_{3}, \ldots, k_{m}\right)+\mathcal{F}\left(k_{3}-1, k_{4}, \ldots, k_{m}\right)
$$

and

$$
\mathcal{F}\left(k_{1}, \ldots, k_{m}\right)=\mathcal{F}\left(k_{1}, \ldots, k_{m-2}, k_{m-1}-1\right)+\mathcal{F}\left(k_{1}, \ldots, k_{m-3}, k_{m-2}-1\right) .
$$

Also,

$$
\mathcal{F}\left(k_{1}, k_{2}\right)=3, \quad \mathcal{F}(k)=2 \text { for } k \geq 0 .
$$

Proof: For $m \geq 4$ the result follows directly from Propositions 2.4 and 2.5 . For $m=3$ the results follows from $\mathcal{F}(1,2,1)=5, \mathcal{F}(1,1,1)=4, \mathcal{F}(1,1)=3, \mathcal{F}(1)=2$, and $\mathcal{F}(0)=2$ which can be easily checked by complete enumeration.

Example 2.1 (cont.) We now use Theorem 2.6 to find the number of fixed points of $\wedge^{2} \vee \wedge \vee^{3} \wedge^{2} \vee$ :

$$
\begin{aligned}
\mathcal{F}(2,1,1,3,2,1) & =\mathcal{F}(1,1,1,2,2,1) \\
& =\mathcal{F}(1-1,1,2,2,1)+\mathcal{F}(1-1,2,2,1) \\
& =\mathcal{F}(1,2,2,1)+\mathcal{F}(2,2,1) \\
& =\mathcal{F}(2-1,2,1)+\mathcal{F}(2-1,1)+\mathcal{F}(2-1,1)+\mathcal{F}(1-1) \\
& =\mathcal{F}(1,2,1)+\mathcal{F}(1,1)+\mathcal{F}(1,1)+\mathcal{F}(0) \\
& =\mathcal{F}(2-1,1)+\mathcal{F}(1-1)+\mathcal{F}(1,1)+\mathcal{F}(1,1)+\mathcal{F}(0) \\
& =\mathcal{F}(1,1)+\mathcal{F}(0)+\mathcal{F}(1,1)+\mathcal{F}(1,1)+\mathcal{F}(0) \\
& =3+2+3+3+2 \\
& =13
\end{aligned}
$$


or

$$
\begin{aligned}
\mathcal{F}(2,1,1,3,2,1) & =\mathcal{F}(1,1,1,2,2,1) \\
& =\mathcal{F}(1,1,1,2,2-1)+\mathcal{F}(1,1,1,2-1) \\
& =\mathcal{F}(1,1,1,2,1)+\mathcal{F}(1,1,1,1) \\
& =\mathcal{F}(1,1,1,2-1)+\mathcal{F}(1,1,1-1)+\mathcal{F}(1,1,1-1)+\mathcal{F}(1,1-1) \\
& =\mathcal{F}(1,1,1,1)+\mathcal{F}(1,1)+\mathcal{F}(1,1)+\mathcal{F}(1) \\
& =\mathcal{F}(1,1,1-1)+\mathcal{F}(1,1-1)+\mathcal{F}(1,1)+\mathcal{F}(1,1)+\mathcal{F}(1) \\
& =\mathcal{F}(1,1)+\mathcal{F}(1)+\mathcal{F}(1,1)+\mathcal{F}(1,1)+\mathcal{F}(1) \\
& =3+2+3+3+2 \\
& =13
\end{aligned}
$$

In this way, Theorem 2.6 provides a recursive formula to compute the number of fixed points of ANDOR networks with chain topology without the need of exhaustive enumeration. We now study 2 especial cases $\mathcal{F}(1,1, \ldots, 1,1)$ and $\mathcal{F}(2,2, \ldots, 2,2)$.

Define $A_{n}=(1, \underbrace{1,1, \ldots, 1,1}_{n \text { times }}, 1)$ and $B_{n}=(2, \underbrace{2,2, \ldots, 2,2}_{n \text { times }}, 2)$. Define the sequences $a_{0}=1, a_{1}=1$, $a_{2}=1$, and $a_{n}=a_{n-2}+a_{n-3}$ for $n \geq 3$ and $b_{0}=1, b_{1}=1$, and $b_{n}=b_{n-1}+b_{n-2}$ for $n \geq 2$. Note that $\left(a_{n}\right)$ is the Padovan sequence and $\left(b_{n}\right)$ is the Fibonacci sequence.

Corollary 2.7. With the definitions above we have $\mathcal{F}\left(A_{n}\right)=a_{n+5}$ and $\mathcal{F}\left(B_{n}\right)=b_{n+3}$ for $n \geq 0$, and the sharp bounds $\mathcal{F}\left(A_{n}\right) \leq \mathcal{F}\left(1, r_{1}, r_{2}, \ldots, r_{n}, 1\right) \leq \mathcal{F}\left(B_{n}\right)$ for all $r_{i} \geq 1$.

Proof: It follows from Theorem 2.6 or Proposition 2.5 using induction.

\section{Infinite and Closed Chain}

In this section we study the cases of AND-OR networks with infinitely many variables and when the topology is a closed chain.

When the AND-OR network has infinitely many variables we have a collection of Boolean functions $f=\left(\ldots, f_{-2}, f_{-1}, f_{0}, f_{1}, f_{2}, \ldots\right)$ such that $f_{i}=x_{i-1} \wedge x_{i+1}$ or $f_{i}=x_{i-1} \vee x_{i+1}$. We can use the same notation of Section 2 and denote consecutive logical operators as $\wedge^{k}$ or $\vee^{k}$, where $k$ could also be $\infty$. Also, we can simply use the exponents to represent the AND-OR network. For example, $(\infty, 1,2, \infty)$ and $\wedge^{\infty} \vee \wedge^{2} \vee^{\infty}$ represent the AND-OR network . . $\wedge \wedge \wedge \vee \wedge \wedge \vee \vee \vee \ldots$, and $(\ldots, 1,1,2,1,1,2,1,1,2, \ldots)$

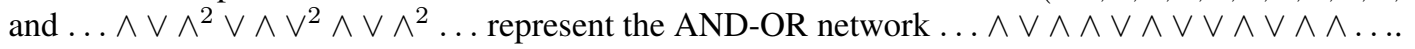

The following theorem allows us to use the results from Section 2 to study AND-OR networks with infinitely many variables.

Theorem 3.1. With the notation above and $k_{i} \geq 1$ we have the following.

$$
\begin{aligned}
\mathcal{F}(\infty) & =2 \\
\mathcal{F}\left(\infty, k_{1}, k_{2}, \ldots, k_{m-1}, k_{m}, \infty\right) & =\mathcal{F}\left(1, k_{1}, k_{2}, \ldots, k_{m-1}, k_{m}, 1\right) \\
\mathcal{F}\left(\infty, k_{1}, k_{2}, k_{3}, \ldots\right) & =\infty \\
\mathcal{F}\left(\ldots, k_{-3}, k_{-2}, k_{-1}, \infty\right) & =\infty \\
\mathcal{F}\left(\ldots, k_{-3}, k_{-2}, k_{-1}, k_{0}, k_{1}, k_{2}, k_{3}, \ldots\right) & =\infty
\end{aligned}
$$


Proof: To prove the first equality we consider the AND-OR network where all logical operators are $\wedge$. If one of the variables is 0 , it follows that all the other variables are also 0 . Similarly, if one of the variables is $\mathbf{1}$, all the other variables are also 1 . Thus, the only fixed points of this AND-OR network are $\mathbf{0}$ and $\mathbf{1}$.

The second equality follows the same approach seen in Proposition 2.4.

To prove the third equality we first observe that $\mathcal{F}\left(\infty, k_{1}, k_{2}, k_{3}, \ldots\right)=\mathcal{F}\left(1, k_{1}, k_{2}, k_{3}, \ldots\right)$. Now, we will show that any fixed point of the AND-OR network $\mathcal{F}\left(1, k_{1}, k_{2}, k_{3}, \ldots, k_{r}\right)$ defines a fixed point of $\mathcal{F}\left(1, k_{1}, k_{2}, k_{3}, \ldots\right)$. Indeed, using the notation of Lemma 2.3. a fixed point of the AND-OR network $\mathcal{F}\left(1, k_{1}, \ldots, k_{r}\right)$ has the form $\mathbf{x}=\left(\mathbf{x}^{0}, \mathbf{x}^{1}, \ldots, \mathbf{x}^{\mathbf{r}}\right)$. Then, denoting $\mathbf{z}=(1,1, \ldots)$ if $\mathbf{x}^{r}=\mathbf{1}$ and $\mathbf{z}=(0,0, \ldots)$ if $\mathbf{x}^{r}=\mathbf{0}$, it follows that $\left(\mathbf{x}^{0}, \mathbf{x}^{1}, \ldots, \mathbf{x}^{\mathbf{r}}, \mathbf{z}\right)$ is a fixed point of $\mathcal{F}\left(1, k_{1}, k_{2}, k_{3}, \ldots\right)$. Since $r$ is arbitrary, $\mathcal{F}\left(1, k_{1}, \ldots, k_{r}\right)$ is not bounded (see Corollary 2.7) and then number of fixed points of $\mathcal{F}\left(1, k_{1}, \ldots\right)$ is $\infty$. The last two equalities are similar.

When the topology of the network is a closed chain, we have the network

$$
f_{1}=x_{n} \diamond_{1} x_{2}, \quad f_{2}=x_{1} \diamond_{2} x_{3}, \quad f_{3}=x_{2} \diamond_{3} x_{4}, \quad \ldots \quad, \quad f_{n-1}=x_{n-2} \diamond_{n-1} x_{n}, \quad f_{n}=x_{n-1} \diamond_{n} x_{1} .
$$

We denote this network as $\left[k_{1}, k_{2}, \ldots, k_{r}\right]$ or any cyclic permutation that groups consecutive logical operators. Thus, the AND-OR network

$$
f_{1}=x_{n} \wedge x_{2}, \quad f_{2}=x_{1} \vee x_{3}, \quad f_{3}=x_{2} \wedge x_{4}, \quad f_{4}=x_{3} \vee x_{5}, \quad f_{5}=x_{4} \vee x_{6}, \quad f_{6}=x_{5} \wedge x_{1},
$$

will not be denoted by $[1,1,1,2,1]$ ("splitting" the first and last $\wedge$ 's), but by $[1,1,2,2],[1,2,2,1]$, $[2,2,1,1]$, or $[2,1,1,2]$ (combining the first and last $\wedge$ 's). This means that $r$ in $\left[k_{1}, k_{2}, \ldots, k_{r}\right]$ will always be an even number or equal to 1 . The number of fixed points will be denoted by $\mathcal{F}\left[k_{1}, k_{2}, \ldots, k_{r}\right]$. The following propositions and theorem allow us to use the results from Section 2 to study AND-OR networks with closed chain topology.

Proposition 3.2. With the notation above, we have that for $k_{i} \geq 1$

$$
\mathcal{F}\left[k_{1}, k_{2}, \ldots, k_{r}\right]=\mathcal{F}\left[\min \left\{2, k_{1}\right\}, \min \left\{2, k_{2}\right\}, \ldots, \min \left\{2, k_{r}\right\}\right] .
$$

Proof: It is analogous to the proof of Proposition 2.4

Proposition 3.3. Consider $k_{i} \geq 1, m \geq 6$, and $l \geq 8$. Then,

$$
\begin{aligned}
\mathcal{F}\left[2, k_{2}, \ldots, k_{m}\right]= & \mathcal{F}\left(k_{2}-1, k_{3}, \ldots, k_{m-1}, k_{m}-1\right)+\mathcal{F}\left(k_{3}-1, k_{4}, \ldots, k_{m-2}, k_{m-1}-1\right), \\
\mathcal{F}\left[1, k_{2}, \ldots, k_{l}\right]= & \mathcal{F}\left(k_{3}-1, k_{4}, \ldots, k_{l-1}-1\right)+\mathcal{F}\left(k_{4}-1, k_{5}, \ldots, k_{l-1}, k_{l}-1\right)+ \\
& \mathcal{F}\left(k_{2}-1, k_{3}, \ldots, k_{l-3}, k_{l-2}-1\right)-\mathcal{F}\left(k_{4}-1, k_{5}, \ldots, k_{l-3}, k_{l-2}-1\right) .
\end{aligned}
$$

Proof: The first equality is analogous to Proposition 2.5. To prove the second equality we use the notation of Lemma 2.3

We have several cases to consider for $k_{l-2}, k_{l-1}, k_{l}, k_{2}, k_{3}$, and $k_{4}$. We focus on the case $k_{l-2}=$ $k_{l-1}=k_{l}=k_{2}=k_{3}=k_{4}=1$ since the other cases are analogous. Note that we want to prove

$$
\begin{aligned}
\mathcal{F}\left[1,1,1,1, k_{5} \ldots, k_{l-3}, 1,1,1\right]= & \mathcal{F}\left(1, k_{5}, \ldots, k_{l-3}, 1\right)+\mathcal{F}\left(k_{5}, \ldots, k_{l-3}, 1,1\right)+ \\
& \mathcal{F}\left(1,1, k_{5}, \ldots, k_{l-3}\right)-\mathcal{F}\left(k_{5}, \ldots, k_{l-3}\right) .
\end{aligned}
$$


The fixed points of the AND-OR network are the solutions of

$$
\begin{aligned}
x_{1} & =x_{n} \wedge x_{2}, \\
x_{2} & =x_{1} \vee x_{3}, \\
x_{3} & =x_{2} \wedge x_{4}, \\
x_{4} & =x_{3} \vee x_{5}, \\
x_{5} & =x_{4} \wedge x_{6}, \\
& \vdots \\
x_{n-3} & =x_{n-4} \wedge x_{n-2}, \\
x_{n-2} & =x_{n-3} \vee x_{n-1}, \\
x_{n-1} & =x_{n-2} \wedge x_{n}, \\
x_{n} & =x_{n-1} \vee x_{1} .
\end{aligned}
$$

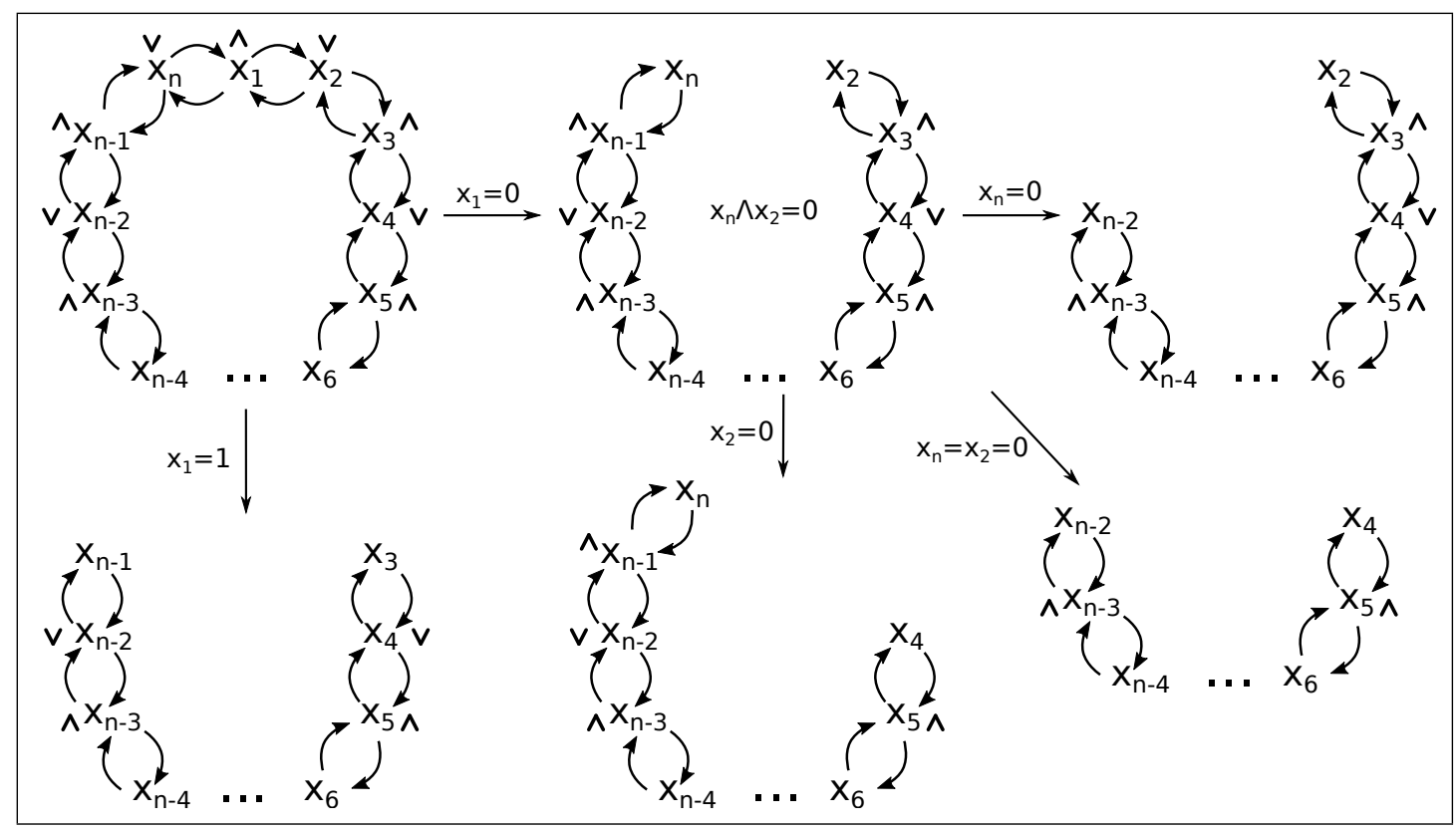

Fig. 3: Idea behind the proof of Proposition 3.3 (logical operators are included for clarity). Considering the case $x_{1}=1$ yields a system of equations that corresponds to a smaller AND-OR network. Considering the case $x_{1}=0$ yields a system of equation that does not correspond to an AND-OR network (due to the equation $x_{n} \wedge x_{2}=0$ ). However, the subcases $x_{n}=0$ and $x_{2}=0$ yield systems of equations that do correspond to smaller AND-OR networks. These two systems have overlapping solutions, so we must also take into consideration the common case $x_{n}=x_{2}=0$ when counting the number of fixed points.

We now consider the cases $x_{1}=1$ and $x_{1}=0$ (see Fig 3 ). The case $x_{1}=1$ yields the system of 
equations

$$
\begin{aligned}
x_{3} & =x_{4}, \\
x_{4} & =x_{3} \vee x_{5}, \\
x_{5} & =x_{4} \wedge x_{6}, \\
& \vdots \\
x_{n-3} & =x_{n-4} \wedge x_{n-2}, \\
x_{n-2} & =x_{n-3} \vee x_{n-1}, \\
x_{n-1} & =x_{n-2},
\end{aligned}
$$

which has $\mathcal{F}\left(1, k_{5}, \ldots, k_{l-3}, 1\right)$ solutions. On the other hand, when we consider $x_{1}=0$ the first equation becomes $x_{n} \wedge x_{2}=0$. We now have 2 subcases: $x_{n}=0$ and $x_{2}=0$. The subcase $x_{n}=0$ yields

$$
\begin{aligned}
x_{2} & =x_{3}, \\
x_{3} & =x_{2} \wedge x_{4}, \\
x_{4} & =x_{3} \vee x_{5}, \\
x_{5} & =x_{4} \wedge x_{6}, \\
& \vdots \\
x_{n-3} & =x_{n-4} \wedge x_{n-2}, \\
x_{n-2} & =x_{n-3},
\end{aligned}
$$

which has $\mathcal{F}\left(1,1, k_{5}, \ldots, k_{l-3}\right)$ solutions. The subcase $x_{2}=0$ yields

$$
\begin{aligned}
x_{4} & =x_{5}, \\
x_{5} & =x_{4} \wedge x_{6}, \\
& \vdots \\
x_{n-3} & =x_{n-4} \wedge x_{n-2}, \\
x_{n-2} & =x_{n-3} \vee x_{n-1}, \\
x_{n-1} & =x_{n-2} \wedge x_{n}, \\
x_{n} & =x_{n-1},
\end{aligned}
$$

which has $\mathcal{F}\left(k_{5}, \ldots, k_{l-3}, 1,1\right)$ solutions. Thus, adding up these 3 numbers we obtain $\mathcal{F}\left(1, k_{5}, \ldots, k_{l-3}, 1\right)+$ $\mathcal{F}\left(k_{5}, \ldots, k_{l-3}, 1,1\right)+\mathcal{F}\left(1,1, k_{5}, \ldots, k_{l-3}\right)$. However, this is not $\mathcal{F}\left[1,1,1,1, k_{5} \ldots, k_{l-3}, 1,1,1\right]$, since the subcases $x_{n}=0$ and $x_{2}=0$ overlap. We need to subtract the number of solutions of the system

$$
\begin{aligned}
x_{4} & =x_{5}, \\
x_{5} & =x_{4} \wedge x_{6}, \\
& \vdots \\
x_{n-3} & =x_{n-4} \wedge x_{n-2}, \\
x_{n-2} & =x_{n-3},
\end{aligned}
$$

which has $\mathcal{F}\left(k_{5}, \ldots, k_{l-3}\right)$ solutions. Then, the result follows.

We now declare some conventions to write Proposition 3.3 more compactly. We define $\mathcal{F}(-1)=1$, $\left(k_{s}-1, \ldots, k_{s}-1\right)=\left(k_{s}-2\right)$, and $\left(k_{s}-1, \ldots, k_{t}-1\right)=(-1)$ for $s>t$. 
Theorem 3.4. With the conventions above, we have that for $m \geq 4$ and $k_{i} \geq 1$

$$
\begin{aligned}
\mathcal{F}\left[2, k_{2}, \ldots, k_{r}\right]= & \mathcal{F}\left(k_{2}-1, k_{3}, \ldots, k_{r-1}, k_{r}-1\right)+\mathcal{F}\left(k_{3}-1, k_{4}, \ldots, k_{r-2}, k_{r-1}-1\right), \\
\mathcal{F}\left[1, k_{2}, \ldots, k_{r}\right]= & \mathcal{F}\left(k_{3}-1, k_{4}, \ldots, k_{r-1}-1\right)+\mathcal{F}\left(k_{4}-1, k_{5}, \ldots, k_{r-1}, k_{r}-1\right)+ \\
& \mathcal{F}\left(k_{2}-1, k_{3}, \ldots, k_{r-3}, k_{r-2}-1\right)-\mathcal{F}\left(k_{4}-1, k_{5}, \ldots, k_{r-3}, k_{r-2}-1\right) .
\end{aligned}
$$

Also,

$$
\begin{aligned}
\mathcal{F}[k] & =2 \text { for } k \geq 3, \\
\mathcal{F}[k, 1] & =2 \text { for } k \geq 2, \\
\mathcal{F}\left[k_{1}, k_{2}\right] & =3 \text { for } k_{1}, k_{2} \geq 2,
\end{aligned}
$$

Proof: The first two equalities follows directly from Proposition 3.2 and 3.3 using the convention declared above. The last 3 equalities follow from Proposition 3.2 and $\mathcal{F}[3]=\mathcal{F}[2,1]=2$ and $\mathcal{F}[2,2]=3$, which can be verified by complete enumeration.

As in Section 2 we now consider the cases $A_{n}=(1, \underbrace{1,1, \ldots, 1,1}_{n \text { times }}, 1)$ and $B_{n}=(2, \underbrace{2,2, \ldots, 2,2}_{n \text { times }}, 2)$. We denote the number of fixed points of the corresponding AND-OR networks with closed chain topology as $\mathcal{F}\left[A_{n}\right]$ and $\mathcal{F}\left[B_{n}\right]$, respectively.

Corollary 3.5. With the notation above we have $\mathcal{F}\left[A_{n}\right]=3 a_{n}-a_{n-2}$ and $\mathcal{F}\left[B_{n}\right]=b_{n+2}+b_{n}$ for $n \geq 2$, and the sharp bounds $\mathcal{F}\left[A_{n}\right] \leq \mathcal{F}\left[k_{0}, k_{1}, \ldots, k_{n}, k_{n+1}\right] \leq \mathcal{F}\left[B_{n}\right]$ for all $r_{i} \geq 1$

Proof: The proof follows from Theorem 3.4 and Corollary 2.7

Example 3.6. We consider

$$
\begin{aligned}
& f_{1}=x_{12} \wedge x_{2}, \quad f_{2}=x_{1} \wedge x_{3}, \quad f_{3}=x_{2} \wedge x_{4}, \quad f_{4}=x_{3} \vee x_{5}, \quad f_{5}=x_{4} \wedge x_{6}, \quad f_{6}=x_{5} \vee x_{7}, \\
& f_{7}=x_{6} \vee x_{8}, \quad f_{8}=x_{7} \vee x_{9}, \quad f_{9}=x_{8} \wedge x_{10}, \quad f_{10}=x_{9} \wedge x_{11}, \quad f_{11}=x_{10} \vee x_{12}, \quad f_{12}=x_{11} \vee x_{1} .
\end{aligned}
$$

We will use Theorems 2.6 and 3.4 for the representations $[3,1,1,3,2,2]$ and $[1,3,2,2,3,1]$ of $f$.

$$
\begin{aligned}
\mathcal{F}[3,1,1,3,2,2] & =\mathcal{F}[2,1,1,2,2,2] \\
& =\mathcal{F}(1-1,1,2,2,2-1)+\mathcal{F}(1-1,2,2-1) \\
& =\mathcal{F}(1,2,2,1)+\mathcal{F}(2,1) \\
& =\mathcal{F}(2-1,2,1)+\mathcal{F}(2-1,1)+\mathcal{F}(2,1) \\
& =\mathcal{F}(1,2,1)+\mathcal{F}(1,1)+\mathcal{F}(2,1) \\
& =\mathcal{F}(2-1,1)+\mathcal{F}(1-1)+\mathcal{F}(1,1)+\mathcal{F}(2,1) \\
& =\mathcal{F}(1,1)+\mathcal{F}(0)+\mathcal{F}(1,1)+\mathcal{F}(2,1) \\
& =3+2+3+3=11
\end{aligned}
$$

$$
\begin{aligned}
\mathcal{F}[1,3,2,2,3,1] & =\mathcal{F}[1,2,2,2,2,1] \\
& =\mathcal{F}(2-1,2,2-1)+\mathcal{F}(2-1,2,1-1)+\mathcal{F}(2-1,2,2-1)-\mathcal{F}(2-2) \\
& =\mathcal{F}(1,2,1)+\mathcal{F}(1,2)+\mathcal{F}(1,2,1)-\mathcal{F}(0) \\
& =\mathcal{F}(1,1)+\mathcal{F}(0)+\mathcal{F}(1,2)+\mathcal{F}(1,1)+\mathcal{F}(0)-\mathcal{F}(0) \\
& =3+2+3+3+2-2=11
\end{aligned}
$$




\section{Conclusion}

Our results provide recursive formulas and sharp bounds for the number of fixed points of AND-OR networks with chain topology. Other work regarding the number of fixed points has focused on bounds with respect to the number of nodes (Aracena et al. 2004). Our results, on the other hand, focus on formulas and bounds with respect to the number of consecutive logical operators. Thus, our results complement previous results.

Our approach can potentially be extended to cases where an AND-OR network has a topology that can be seen as the "combination" of open chains. Then, the number of fixed points of the original AND-OR network will be given by the inclusion-exclusion principle in terms of the number of fixed points of the AND-OR networks with open chain topology. Indeed, Theorem 3.4 shows how our approach can be used in such cases.

\section{References}

Z. Agur, A. Fraenkel, and S. Klein. The number of fixed points of the majority rule. Discrete Math., 70 (3):295-302, 1988. ISSN 0012-365X.

T. Akutsu, S. Kuhara, O. Maruyama, and S. Miyano. A system for identifying genetic networks from gene expression patterns produced by gene disruptions and overexpressions. Genome Inform., 9:151-160, 1998.

R. Albert and H. Othmer. The topology of the regulatory interactions predicts the expression pattern of the segment polarity genes in Drosophila melanogaster. J. Theor. Biol., 223:1-18, 2003.

J. Aracena. Maximum number of fixed points in regulatory boolean networks. Bulletin of Mathematical Biology, 70(5):1398-1409, 2008. URL DOI:10.1007/s11538-008-9304-7

J. Aracena, J. Demongeot, and E. Goles. Fixed points and maximal independent sets in AND-OR networks. Discrete Appl. Math., 138(3):277-288, 2004. ISSN 0166-218X.

A. Jarrah, B. Raposa, and R. Laubenbacher. Nested canalyzing, unate cascade, and polynomial functions. Physica D: Nonlinear Phenomena, 233(2):167 - 174, 2007. ISSN 0167-2789. doi: 10.1016/ j.physd.2007.06.022. URL http://www.sciencedirect.com/science/article/pii/ S0167278907002035

A. Jarrah, R. Laubenbacher, and A. Veliz-Cuba. The dynamics of conjunctive and disjunctive Boolean network models. Bull. Math. Bio., 72(6):1425-1447, 2010. doi: 10.1007/s11538-010-9501-z.

L. Mendoza and I. Xenarios. A method for the generation of standardized qualitative dynamical systems of regulatory networks. Theoretical Biology and Medical Modelling, 3(1):13, 2006. ISSN 1742-4682. doi: 10.1186/1742-4682-3-13. URL http://www.tbiomed.com/content/3/1/13

D. Murrugarra and R. Laubenbacher. Regulatory patterns in molecular interaction networks. Journal of Theoretical Biology, 288(0):66 - 72, 2011. ISSN 0022-5193. doi: 10.1016/j.jtbi.2011.08.015. URL http://www.sciencedirect.com/science/article/pii/s0022519311004103. 
A. Veliz-Cuba and R. Laubenbacher. On the computation of fixed points in Boolean networks. Journal of Applied Mathematics and Computing, 39(1-2):145-153, 2011.

A. Veliz-Cuba and B. Stigler. Boolean models can explain bistability in the lac operon. J. Comput. Biol., 18(6):783-794, 2011.

A. Veliz-Cuba, K. Buschur, R. Hamershock, A. Kniss, E. Wolff, and R. Laubenbacher. AND-NOT logic framework for steady state analysis of Boolean network models. Applied Mathematics and Information Sciences, 7(4):1263-1274, 2013.

A. Veliz-Cuba, A. Kumar, and K. Josić. Piecewise linear and Boolean models of chemical reaction networks. Bulletin of Mathematical Biology, 76(12):2945-2984, 2014.

A. Veliz-Cuba, B. Aguilar, and R. Laubenbacher. Dimension reduction of large sparse AND-NOT network models. Electronic Notes in Theoretical Computer Science, 316:83-95, 2015. 